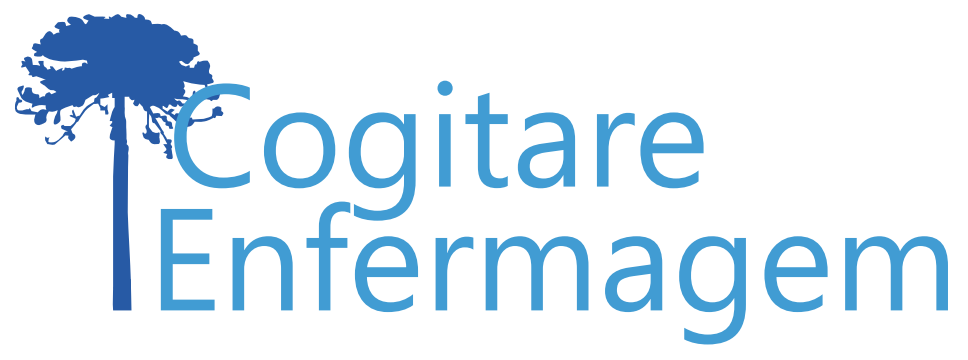

\title{
RASTREIO E ENCAMINHAMENTO DE CASOS DE VIOLÊNCIA CONTRA A MULHER POR ENFERMEIRAS NA ESTRATÉGIA SAÚDE DA FAMÍLIA
}

Josy Cárolen Vieira de Lima ${ }^{1}$, Renata Clemente dos Santos ${ }^{2}$, Jessyka Chaves da Silva ${ }^{3}$, Rebeca de Sousa Costa da Silva ${ }^{4}$, Cláudia Maria Ramos Medeiros Souto5 , Rafaella Queiroga Souto6, Gleicy Karine Nascimento de Araújo ${ }^{7}$

\section{RESUMO}

Objetivo: compreender como se realiza o rastreamento e encaminhamento de casos de violência contra a mulher por enfermeiras da Estratégia de Saúde da Família do interior paraibano.

Método: estudo transversal, qualitativo, participando sete enfermeiras atuantes na Estratégia de Saúde da Família, vinculadas ao distrito sanitário VI de saúde do município de Campina Grande, Paraíba. A coleta ocorreu em setembro de 2018 e foi realizada análise de conteúdo de Bardin.

Resultados: emergiram três categorias: I - Conceitos de violência (29,3\%); II - Rastreio de casos de violência (53,7\%); III - Encaminhamento de casos de violência (17,1\%). Observou-se as palavras "agressão física",' delegacia da mulher", "assistente social", "psicológico", "denunciar", "lembrar", "sofrer", "físico".

Conclusão: as enfermeiras compreendem a violência e a consulta ginecológica serve para identificar os casos. A pesquisa esclarece pontos de fragilidade dos serviços de saúde que dificultam o combate à violência e demonstram a necessidade da qualificação da equipe.

DESCRITORES: Violência contra a Mulher; Violência de Gênero; Estratégia Saúde da Família; Enfermagem; Enfermagem Forense.

\section{COMO REFERENCIAR ESTE ARTIGO:}

Lima JCV de, Santos RC dos, Silva JC da, Silva R de SC da, Souto CMRM, Souto RQ, et al. Rastreio e encaminhamento de casos de violência contra a mulher por enfermeiras na estratégia saúde da família. Cogitare enferm. [Internet]. 2020 [acesso em "colocar data de acesso, dia, mês abreviado e ano"]; 25. Disponível em: http://dx.doi.org/10.5380/ce.v25i0.65579.

\section{(c) (1)}

Este obra está licenciado com uma Licença Creative Commons Atribuição 4.0 Internacional.

\section{${ }^{1}$ Enfermeira. Centro Universitário Unifacisa. Campina Grande, PB, Brasil.}

${ }^{2}$ Enfermeira. Mestre em Enfermagem. Docente de Enfermagem do Centro Universitário Unifacisa. Campina Grande, PB, Brasil. $(8$

${ }^{3}$ Enfermeira. Mestranda em Enfermagem. Universidade de Pernambuco. Campina Grande, PB, Brasil. $\odot$

${ }^{4}$ Discente de Enfermagem. Centro Universitário Facisa. Campina Grande, PB, Brasil. $\bigcirc$

${ }^{5}$ Enfermeira. Doutora em Enfermagem. Docente de Enfermagem da Universidade Federal da Paraíba. João Pessoa, PB, Brasil. $(0$

${ }^{6}$ Enfermeira. Pós-doutora em Enfermagem. Docente de Enfermagem da Universidade Federal da Paraíba. João Pessoa, PB, Brasil. $($ )

${ }^{7}$ Enfermeira. Mestranda em Enfermagem. Universidade Federal da Paraíba. João Pessoa, PB, Brasil. 


\title{
TRACKING AND REFERRAL BY NURSES IN THE FAMILY HEALTH STRATEGY OF VIOLENCE CASES AGAINST WOMEN
}

\begin{abstract}
Objective: understand how the tracking and referral of cases of violence against women by nurses of the Family Health Strategy of the upstate Paraiba are carried out.

Method: a transversal, qualitative study, with the participation of seven nurses working in the Family Health Strategy, linked to the VI health district of the municipality of Campina Grande, Paraiba. The collection took place in September 2018 and a content analysis of Bardin was performed.

Results: three categories have emerged: I - Concepts of violence (29.3\%); II - Tracking of violence cases (53.7\%); III - Referral of violence cases (17.1\%). The words "physical aggression", "woman's police station", "social worker", "psychological", "denounce", "remember", "suffer", "physical" were observed.

Conclusion: nurses understand violence and the gynecological consultation serves to identify cases. The research clarifies points of the fragility of health services that make it difficult to fight violence and demonstrate the need for team qualification.
\end{abstract}

DESCRIPTORS: Violence against Women; Gender Violence; Family Health Strategy; Nursing; Forensic Nursing.

\section{RASTREO Y DERIVACIÓN DE CASOS DE VIOLENCIA CONTRA LA MUJER POR ENFERMERAS EN EL MARCO DE LA ESTRATEGIA DE SALUD DE LA FAMILIA}

\section{RESUMEN:}

Objetivo: comprender cómo se realiza el rastreo y la derivación de casos de violencia contra la mujer por parte de enfermeras en el marco de la Estrategia de Salud de la Familia del interior del Estado de Paraíba.

Método: estudio transversal, cualitativo, en el que participaron siete enfermeras de la Estrategia de Salud da la Familia, vinculadas al distrito sanitario de salud VI del municipio de Campina Grande, Paraíba. La recolección de datos tuvo lugar en septiembre de 2018 y se realizó el análisis de contenido de Bardin.

Resultados: surgieron tres categorías: I - Conceptos de violencia (29,3\%); II - Rastreo de casos de violencia (53,7\%); III - Derivación de casos de violencia (17,1\%). Se observó el uso de las palabras "agresión física", "comisaría de la mujer", "asistente social", "psicológico", "denunciar", "recordar", "sufrir", "físico".

Conclusión: las enfermeras comprenden la violencia y la consulta ginecológica sirve para identificar los casos. La investigación aclara puntos de fragilidad de los servicios de salud que dificultan el combate a la violencia y demuestran la necesidad de contar con equipo cualificado.

DESCRIPTORES: Violencia contra la Mujer; Violencia de Género; Estrategia de Salud de la Familia; Enfermería; Enfermería Forense. 
Desde o início da década de 70, a violência contra a mulher (VCM) vem ganhando atenção e mobilização. O problema inclui diferentes formas de violência, seja ela física, psicológica, moral, sexual ou patrimonial. Sua ocorrência apresenta uma perspectiva baseada na desigualdade de gênero mediante a construção social de papéis ${ }^{(1-2)}$.

A VCM é definida como qualquer ato baseado no gênero feminino, que cause morte, danos físicos irreversíveis, sexual ou psicológico à mulher. Entende-se que é um componente concreto de violação dos direitos humanos, levando a ser caracterizado como uma problemática de cunho judicial e de saúde pública ${ }^{(3)}$.

A violência pode gerar problemas no estado psíquico e na integridade física da vítima. Sendo assim, as formas mais comuns de serem observadas pelos profissionais da saúde, principalmente quando relacionado à estratégia de saúde da família, são as lesões corporais, em músculos, ossos, na região ocular, mordidas, além dos problemas comportamentais como tirar de casa à força, desmoralizar, arrastar, sofrer ameaças, utilização de palavras de baixo calão e doenças decorrentes de formas de negligência. Tal ato pode gerar complicações tanto para a vítima, quanto para os familiares que estão inseridos naquele contexto pela vivência em ambiente transtornado com violência(4-5).

Os profissionais da área de saúde possuem significativa importância para a identificação desse agravo, pois o adoecimento decorrente de violência faz com que a vítima recorra aos serviços de saúde. Estes, por sua vez, precisam estar preparados para oferecer-lhes um atendimento integral, que estimule seu empoderamento, a quebra do ciclo da violência e a minimização dos impactos causados por ela. Cabe-lhes ainda conhecer a articulação da rede de assistência para conduzir e realizar os encaminhamentos corretos da vítima, para que seja alcançada a resolutividade do problema ${ }^{(6)}$.

Em situações de violência física, existem condutas a serem tomadas após ocorrido o evento, como coleta de vestígios, acolhimento da vítima, preenchimento da ficha de notificação compulsória e o relatório para ser encaminhado às autoridades policiais antes que os vestígios desapareçam. $\mathrm{O}$ atendimento deve zelar pela humanização e respeito à vontade da vítima, e a enfermeira deve orientá-la. Os familiares devem ser engajados nos serviços especializados do serviço para que a vítima e familiares sintam segurança e para que possa viabilizar uma atenção integral(6-8).

Nesta perspectiva, é importante que a enfermeira da ESF tenha habilidade para rastrear e encaminhar casos de VCM. Para isso, o presente estudo objetivou compreender como se realiza o rastreamento e encaminhamento de casos de VCM por enfermeiras da ESF do interior paraibano.

\section{MÉTODO}

Trata-se de um estudo transversal, descritivo e exploratório com abordagem qualitativa. A pesquisa foi desenvolvida no distrito sanitário VI de Campina Grande-PB, que conta com treze equipes da estratégia de saúde da família, que se localizam em Malvinas, Rocha Cavalcante, Catolé De Boa Vista, Jardim Verdejante, Paus Brancos e Três irmãs.

A população foi composta por treze profissionais da enfermagem que atuam nas ESF do referido distrito e a amostra foi aleatória, não probabilística por conveniência, totalizando sete enfermeiras.

Foram incluídas enfermeiras que atuavam na ESF vinculada ao distrito sanitário VI de saúde do município de Campina Grande-PB, e foram excluídas aqueles que se encontravam em afastamento do trabalho por férias, atestado médico, licença maternidade ou que não 
se encontraram no serviço após três visitas sem êxito de contato com o enfermeiro(a) da unidade.

Foram utilizados dois instrumentos para coleta elaborados pelos pesquisadores, a fim de atender aos objetivos propostos. O primeiro é um questionário contendo questões relativas à caracterização da amostra e o segundo contempla uma entrevista semiestruturada relativa a conceitos, o exercício da enfermagem na ESF e encaminhamentos em casos confirmados VCM, sendo as questões descritas no Quadro 1.

Quadro 1 - Questões utilizadas no instrumento de coleta de dados do estudo. Campina Grande, PB, Brasil, 2018

1- O que a Sr./Sr. ${ }^{a}$ compreende por violência contra a mulher? Para o Sr./Sr. ${ }^{a}$ o que pode ser considerado como violência?

2- Como é realizado o rastreamento de casos de violência contra a mulher na unidade?

3- Você poderia nos descrever quais os sinais o Sr./Sr. observa na avaliação a mulher que indiquem violência?

4- A Sr./Sr. ${ }^{a}$ durante o exercício da enfermagem na unidade já se deparou com algum caso de violência contra a mulher? Se sim, você pode nos relatar como se desenvolveu a abordagem e o encaminhamento dela nos serviços, se não, o Sr./Sr. ${ }^{a}$ sabe nos relatar os pontos de referência para o encaminhamento em possíveis casos de violência contra mulher?

Foi realizado contato prévio com as enfermeiras que atuam nas ESFs do distrito sanitário VI e nesse momento, foi feito o convite para contribuírem com a pesquisa como participantes da amostra. Em seguida foi realizado o agendamento da coleta de dados de acordo com a conveniência de data e hora do pesquisado.

Após o agendamento, a pesquisadora se deslocou para ESF na qual a profissional atua e esta foi convidada a deslocar-se para um ambiente silencioso e privado, nesse momento lhes foram expostos os objetivos da pesquisa, seus benefícios e contribuição acadêmica, bem como seus possíveis riscos.

As participantes responderam um questionário sociodemográfico, a uma entrevista conduzida por um roteiro contendo questões norteadoras, e gravação de voz, no formato MP3 compondo estes o material empírico coletado para a investigação.

Os dados foram transcritos e seu corpus textual foi submetido ao programa IRAMUTEC (Interface de R pourles Analyses Multidimensionnelles de Textes et de Questionnaires), uma ferramenta de informática a qual proporciona diferentes tipos de análise de corpus textuais ${ }^{(9)}$. O presente estudo adotou a nuvem de palavras e a Classificação Hierárquica Descendente (CHD).

A nuvem de palavras é expressa em forma de gráfico com palavras-chaves presentes no texto mediante a realização dos agrupamentos das palavras em função da sua frequência de ocorrência no texto(9). Já a CHD é uma análise em forma de classes segmentadas do texto; a partir de uma lógica estatística, classifica e organiza os vocabulários com o mesmo contexto, mas cada um nas suas diferentes classes. Já a nuvem trata-se de um conjunto de palavras organizadas em diferentes tamanhos, que a partir de um determinado escore estatístico, dá ênfase às palavras mais marcantes do corpus textual, deixando-as com a fonte maior e centralizada na nuvem ${ }^{(10)}$. 
A formação das classes possibilitou a formação das categorias analíticas, de forma que os trechos foram analisados à luz da Análise de Conteúdo(11), que analisa os dados empíricos alicerçados nos seguintes eixos: pré-análise, descrição analítica e interpretação inferencial. A pré-análise é a escolha dos documentos escolhidos com objetividade e referencial teórico para a construção do texto. A descrição analítica destina-se à exploração aprofundada do material coletado e a interpretação inferencial tem como base classificar e organizar os elementos coletados, por meio de diferenças e semelhanças, seguindo critérios a fim de concluir o conteúdo(11).

Esta pesquisa foi aprovada pelo Comitê de Ética e Pesquisa do Centro de Ensino Superior e Pesquisa - CESED sob o número do parecer 2.804.989.

\section{RESULTADOS}

Mediante a análise do corpus proveniente das entrevistas, houve 2.368 ocorrências de palavras, difundidas em 466 formas, destas 200 ativas e 260 suplementares, e a frequência de formas ativas foi de 3:49. Houve retenção de $60 \%$ do corpus, obtendo como produto a nuvem de palavras na Figura 1 e os dados da Tabela 1.

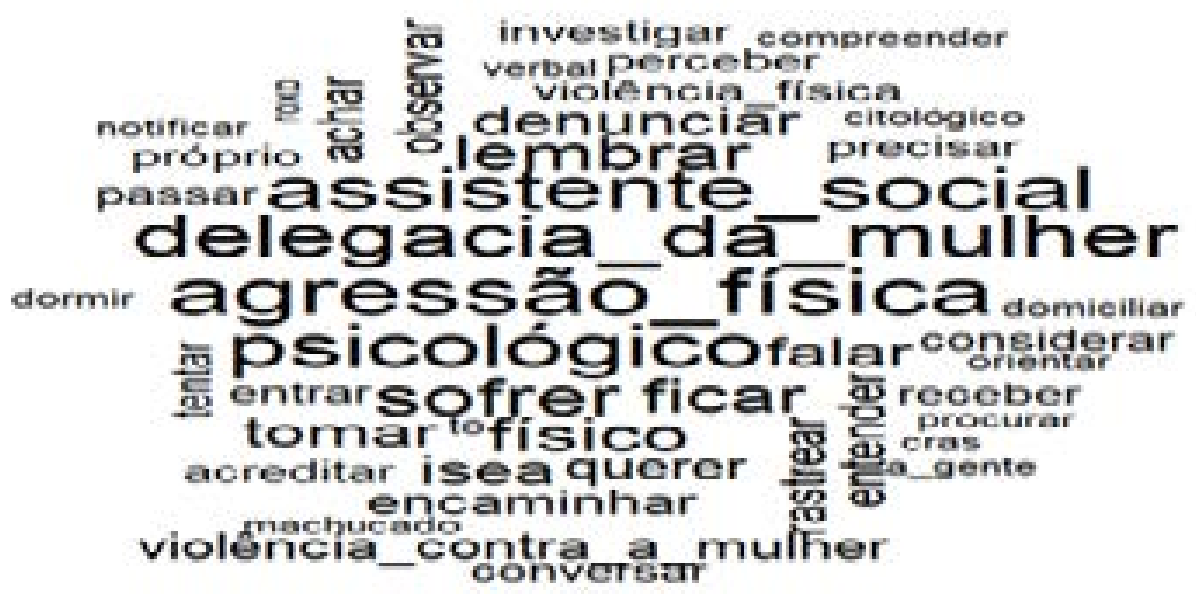

Figura 1 - Nuvem de Palavras proveniente do corpus textual. Campina Grande, PB, Brasil, 2018

Tabela 1 - Classificação Hierárquica Descendente. Campina Grande, PB, Brasil, 2018

Conceito de Violência (29,3\%)

\begin{tabular}{lcc}
\hline Agressão Física & 20,4 & $<0,0001$ \\
\hline Físico & 16,99 & $<0,0001$ \\
\hline Violência contra a mulher & 13,76 & 0,000 \\
\hline Psicológico & 4,75 & 0,02 \\
\hline Rastreamento de Casos de violência (53,7\%) & $\%$ & p-valor \\
\hline Sofrer & 21,8 & $<0,0001$ \\
\hline
\end{tabular}




\begin{tabular}{lcc}
\hline Conversar & 13,3 & 0,000 \\
\hline Mulher & 11,92 & 0,001 \\
\hline Denunciar & 10,5 & 0,000 \\
\hline Conversar & 5,38 & 0,02 \\
\hline Encaminhamento de casos de Violência (17,1\%) & $\%$ & p-valor \\
\hline Delegacia da Mulher & 34,82 & $<0,0001$ \\
\hline ISEA & 27,66 & $<0,0001$ \\
\hline Receber & 15,72 & $<0,0001$ \\
\hline CRAS & 15,72 & $<0,0001$ \\
\hline Encaminhar & 5,62 & 0,017 \\
\hline Trauma & 5,62 & 0,018
\end{tabular}

De acordo com a análise léxica acima, foi possível observar que as palavras "agressão física", "delegacia da mulher", "assistente social", "psicológico", "denunciar", "lembrar", "sofrer", "físico" foram palavras-chave que tiveram grande expressividade nos discursos das enfermeiras colaboradoras do estudo, que corroboram com as classes da Classificação Hierárquica Descendente demonstrada pela Tabela 1.

A partir dos achados e da ocorrência de palavras em cada classe, obteve-se três categorias analíticas, que receberam análise temática discutida a seguir: Categoria 1 Conceito de Violência (29,3\%); Categoria 2 - Rastreamento de casos de violência (53,7\%); Categoria 3 - Encaminhamento de casos de violência $(17,1 \%)$.

\section{Categoria 1 - Conceitos de violência (29,3\%)}

Ao serem questionadas sobre o conceito de violência contra a mulher, foi possível observar o que as enfermeiras da ESF compreendem como a violência contra a mulher, pela conceituação expressa nas falas a seguir:

Não só o fato da agressão física em si... Mas assim, a agressão verbal, a agressão psicológica, então tudo aquilo que venha ferir a mulher não só com a agressão física em si eu entendo que seja uma violência contra a mulher. Violência é agressão física, verbal e psicológica. (E1)

Violência é qualquer forma de agressão física, moral ou verbal, por qual aquela mulher passe. Violência pode ser verbal, onde aquela pessoa é denegrida moralmente ou ridicularizada, ou exposta e pode ser também a violência física em si. (E2)

Os relatos das enfermeiras expressam o significado de VCM de modo genérico, diversificando suas formas de apresentação conforme as palavras chaves da Figura 1, a partir da análise da Nuvem de Palavras, a qual apresenta termos chaves com maior expressividade, dentre estes, acometimento físico, psicológico e moral.

\section{Categoria 2 - Rastreio de casos de violência (53,7\%)}

Quando levantado o questionamento de como é realizado o rastreamento de casos de violência contra a mulher na unidade, e quais os sinais e sintomas observados na avaliação da mulher que indiquem violência, as colaboradoras elencam que a consulta de enfermagem da mulher na ESF é um momento propício para investigação de sinais de 
violência.

Tem algumas né, que vem assim com opressão, que o marido assim... o que eu tenho mais hábito de conversar com as mulheres é na citologia, então na citologia, às vezes a gente identifica assim, maridos meio que forçam as mulheres a terem relação sexual mesmo sem o consentimento delas né isso? (E3)

Foi justamente quando eu fui fazer o citológico que eu vi que ela estava com o corpo com umas manchas roxas, aí eu fui conversando e ela desconversou na hora, aí disse que não era nada, que tudo que ela batia, ela ficava assim, mas aí depois quando ela veio apresentar o exame, ela disse que realmente tinha sido[...]. (E7)

Observa-se que o momento do citológico é observado como estratégico para realização do rastreamento de situações de violência. As participantes elencaram ain da que é nesse momento que é possível observar timidamente alguns sinais e sintomas comuns em casos de ocorrência de violência.

[...]quando a mulher chega com manchas no corpo que não indicam queda, manchas principalmente no mesmo local, aí ela diz que foi queda, que não é manchas de queda nesse caso[...]. (E2)

A gente investiga se está com algum problema na familia, aquelas mulheres que chegam bem nervosas, aí a gente começa a investigar se tem algum problema na família, pra ver se ela fala né, que está sofrendo algum tipo de agressão. (E7)

Hematomas, manchas, retraimento da mulher na consulta e nervosismo foram sinais e sintomas elencados pelas colaboradoras do estudo. Nesse sentido, ainda na mesma questão norteadora, foi possível observar um conhecimento limitado de execução do rastreio de casos de violência.

Descobrir né, aí um caso desse geralmente eu passo pra assistente social, aí a assistente social é quem encaminha mesmo, mas eu, eu... honestamente hoje eu não sei, sei não. (E7)

[...] busca ativa, às vezes um agente de saúde ou outro que venha, que diga, olha aquela mulher ali sofreu, aí a gente aciona o serviço social e a gente vai junto com o serviço social, mas geralmente a gente espera mais a demanda vir, sabe [...]. (E3)

Observou-se que, na prática assistencial, as enfermeiras delegam ao assistente social os casos de violência bem como a não realização da busca ativa de novos casos na comunidade.

\section{Categoria 3 - Encaminhamento de casos de violência (17,1\%)}

Foi questionado qual o encaminhamento realizado pelo profissional para mulheres vítimas de violência e quais os pontos de referência. Como resultado, o ISEA, CRAS e delegacia da mulher ficaram mais explícitos nos discursos, porém sem articulação sólida entre eles, conforme observado nas falas adiante.

O CRAS né, o centro de referência de saúde da mulher, o ISEA quando as mulheres vítimas de violência sexual a gente consegue identificar... é... e a delegacia da mulher. (E2)

Delegacia da mulher, o hospital né... é, geralmente é o hospital de trauma, que é como os dois casos que a gente já pegou aqui foram pro hospital de trauma e fora disso eu não sei... só o serviço de assistência social daqui e também o CRAS, o CRAS que tem o serviço de atendimento psicológico e social. (E4)

Desde década de 1980, a integralidade da atenção à saúde da mulher em todas as fases da vida assegurada pela Constituição Federal e a efetiva criação de importantes órgãos antes inexistentes trouxeram avanços e benefícios para as mulheres em vários setores 
sociais. Sua importância existe nos compromissos assumidos, obrigações governamentais e na formulação de políticas públicas nos âmbitos nacional, estadual e municipal.

\section{DISCUSSÃO}

A VCM é um fenômeno de preocupação na área da saúde e judicial, visto que os seus impactos envolvem danos individuais e coletivos, alterando os padrões de morbimortalidade de uma comunidade, exigindo assim a elaboração de ações e políticas que atendam a completude da mulher. $\hat{E}$ fundamental que os profissionais da enfermagem participem como protagonistas dessas ações e estejam aptos para conceituar a violểncia, identificar casos e realizar seus devidos encaminhamentos de acordo com a demanda trazida pela mulher ${ }^{(12)}$.

No presente estudo, o conceito de violência relatado pelas profissionais de enfermagem leva em consideração aspectos de natureza física, psicológica e social. Achados semelhantes foram observados em pesquisa desenvolvida em Jataí-GO, em que os profissionais de enfermagem conceituaram a violência de forma abrangente, além das queixas físicas ou biológicas, incluindo agressão física, verbal, psicológica, abusos morais e patrimoniais ${ }^{(13)}$.

Intervir no fenômeno da violência perpassa a compreensão conceitual dele, pois exige do profissional a compreensão da sua complexidade para então reconhecer a demanda, que pode exceder a capacidade de resolução em apenas um serviço(12). As enfermeiras entendem a necessidade de uma abordagem integral à mulher para que seja possível observar linguagem verbal e não verbal, que será fundamental na identificação da violência. A Unidade de saúde é o espaço no qual as mulheres buscam cuidados e serviços de prevenção e promoção de saúde, possibilitando a formação de vínculo de respeito e confiança com os profissionais ${ }^{(14)}$.

Assim, a assistência à mulher vítima de violência necessita mais do que o desenvolvimento de habilidades técnicas dos profissionais de saúde, é preciso extrapolar os modelos assistenciais focados na doença e desenvolver ações de cuidado humanizado e que observem o caráter social que envolve o fenômeno da violência doméstica(15).

A consulta de enfermagem é o momento estratégico para identificar situações de violência, em que o profissional deve utilizar estratégias para esclarecimento da situação, a fim de traçar um plano de cuidado adequado, respeitando as necessidades da vítima ${ }^{(4)}$. Esse momento foi também elencado pelas colaboradoras do presente estudo como o mais adequado para identificação de casos de VCM.

É atribuição do enfermeiro reconhecer, durante a consulta de enfermagem, sinais e sintomas que tenham relação direta ou indireta com a violência, como hematomas, queimaduras, mordidas ou lesões cutâneas. A busca pelo serviço de saúde pelas mulheres comumente só acontece em situações mais graves, cabendo então ao profissional da saúde incluir ações que possam identificar precocemente essas situações ${ }^{(7)}$.

Associado a isso, a formação do enfermeiro ao nível de graduação deve contemplar componentes curriculares com abordagem e reflexão dos aspectos comprometedores vivenciados pelas mulheres em situação de violência, incluindo o conhecimento de políticas, programas, a Lei Maria da Penha e redes de apoio voltadas à prevenção, combate à violência e atenção integral. A formação integral daria qualificação profissional, permitindo um acolhimento e atendimento a essas vítimas, propiciando ao longo da formação a construção de conhecimento científico sobre o tema ${ }^{(16)}$.

Essa aparente carência na formação pode justificar a terceirização de atribuições ao assistente social que compõe a equipe de saúde, como a visita domiciliar, escuta qualificada e outros recursos usualmente utilizados para observar e avaliar o contexto de 
vida dos moradores, que podem orientar e direcionar um cuidado através das informações colhidas ${ }^{(17)}$. A demanda de busca ativa de casos de violência na comunidade foi elencada pelos enfermeiros como atribuição do assistente social da equipe.

Aguardar que a vítima venha até o serviço para fazer a denúncia só aumenta os riscos de lesões e danos causados pela agressão. A execução da busca ativa por enfermeiros possibilita a realização de práticas assistenciais mais efetivas, pois se trata de uma valiosa ferramenta no combate à violência, dando-lhe maior visibilidade e possibilitando a prevenção da ocorrência de novos $\operatorname{casos}^{(18)}$.

Estudo realizado em Salvador-BA elencou problemas de socialização entre os diferentes serviços de apoio à mulher em situação de violência. Os serviços que auxiliam no enfrentamento do agravo funcionam ainda como um ponto de comunicação e apontamento de dúvidas entre os profissionais envolvidos na assistência ${ }^{(12)}$. Os profissionais da pesquisa conseguiram elencar alguns serviços de atendimento à vítima, porém sem adequada articulação de rede entre eles, demonstrando-nos na rede de atendimento e, então, deficiência na assistência de enfermagem e de saúde para esse público.

A carência no encaminhamento de mulheres vítimas de violência não se explicita apenas entre os profissionais da enfermagem, estendendo-se aos demais que lhe ofertam assistência. Uma pesquisa realizada no interior do Sudoeste da Bahia apontou que os entrevistados pressupõem que o encaminhamento deve se dar apenas para a delegacia da mulher, sugerindo assim que o problema da violência é apenas da segurança pública. Uma pequena parcela refere-se à rede de atendimento apenas ao setor de saúde, sugerindo, portanto, que esses participantes desconhecem o verdadeiro conceito de intersetorialidade ${ }^{(19)}$.

Durante a coleta de dados, grande parte das profissionais procuradas para a pesquisa relataram uma sobrecarga de trabalho na UBS, demanda grande de pacientes, questões internas e outras não se encontravam no serviço, dificultando o acesso e tornando pequeno o número de enfermeiras participantes da pesquisa, sendo assim, uma limitação do presente estudo.

\section{CONSIDERAÇÕES FINAIS}

Oestudo revelou que os profissionais da enfermagem conhecem conceitosimportantes sobre a temática da violência, porém limitado do ponto de vista de encaminhamento e rastreio da sua ocorrência. A fala das colaboradoras demonstra que não há realização do rastreio do fenômeno, pois terceirizam a atribuição de busca ativa para o serviço social, ou aguardam que as denúncias cheguem até a UBS para tomada de decisões, demonstrando assim carência na execução de ações de promoção e prevenção do agravo.

Observou-se ainda que alguns profissionais desconhecem boa parte dos serviços de referência para encaminhamento dos casos de violência que são apresentados na unidade, ou os conhece de maneira fragmentada sem a interlocução necessária da rede de apoio, dificultando o combate do fenômeno e o rompimento com o ciclo violento.

A pesquisa esclarece pontos de fragilidade existentes nos serviços de saúde que dificultam o combate à violência, assim como a exata compreensão da sua magnitude enquanto problema de saúde pública. Demonstra a necessidade da qualificação da equipe de enfermagem concernente ao rastreio e encaminhamento adequado dos casos na ESF.

Espera-se que a partir deste trabalho sejam desenvolvidos outros estudos, abrangendo outras áreas do município estudado, assim como a elaboração de instrumentos e fluxogramas de auxiliem a prática da ESF, a fim de ofertar maior visibilidade aos casos de violência e tornar possível o enfrentamento desse fenômeno, bem como a busca por estratégias e soluções para os problemas levantados. 
1. Leite FMC, Amorim MHC, Wehrmeister FC, Gigante DP. Violence against women, Espírito Santo, Brazil. Rev. Saúde Pública [Internet]. 2017 [acesso em 05 dez 2018]; 51. Disponível em: http://dx.doi. org/10.1590/s1518-8787.2017051006815.

2. Zuchi CZ, Silva EB da, Costa MC da, Arboit J, Fontana DRG, Honnef F, Heisler ED. Violência contra as mulheres: concepção de profissionais da estratégia de saúde da família acerca da escuta. REME [Internet]. 2018 [acesso em 05 dez 2018]; 22. Disponível em: http://www.dx.doi.org/10.5935/1415-2762.20180015.

3. Comissão Interamericana de Direitos Humanos. Convenção interamericana para prevenir, punir e erradicar a violência contra a mulher. [Internet] Convenção de Belém do Pará; 1994 [acesso em 12 dez 2019]. Disponível em: http://www.cidh.org/basicos/portugues/m.belem.do.para.htm.

4. Freitas RJM de, Sousa VB de, Costa e Cruz T da S, Feitosa RMM, Monteiro ARM, Moura NA de. Atuação dos enfermeiros na identificação e notificação dos casos de violência contra a mulher. HU Revista [Internet]. 2017 [acesso em 22 dez 2018]; 43(2). Disponível em: https://periodicos.ufjf.br/index.php/ hurevista/article/view/2585.

5. Amarijo CL, Gomes VL de O, Gomes AMT, Fonseca AD da, Silva CD. Representação social de profissionais de enfermagem acerca da violência doméstica contra a mulher: abordagem estrutural. Rev. enferm. UERJ. [Internet]. 2017 [acesso em 28 dez 2018]; 25. Disponível em: https://doi.org/10.12957/ reuerj.2017.23648.

6. Guimarães RCS, Soares MC da S, Santos RC dos, Moura JP, Freire TVV, Dias MD. Impacto na autoestima de mulheres em situação de violência doméstica atendidas em Campina Grande, Brasil. Rev Cuid. [Internet]. 2018 [acesso em 29 dez 2018]; 9(1). Disponível em: http://dx.doi.org/10.15649/cuidarte. v9i1.438.

7. Mandelbaum B, Schraiber LB, D'Oliveira AFP. Violência e vida familiar: abordagens psicanalíticas e de gênero. Saúde soc. [Internet]. 2016 [acesso em 02 dez 2019]; 25(2). Disponível em: https://doi. org/10.1590/S0104-12902016145768.

8. Doku DT, Asante KO. Women's approval of domestic physical violence against wives: analysis of the Ghana demographic and health survey. BMC womens health. [Internet]. 2015 [acesso em 10 jan 2019]; 15(1). Disponível em: https://doi.org/10.1186/s12905-015-0276-0.

9. Souza MAR de, Wall ML, Thuler AC de MC, Lowen IMV, Peres AM. O uso do software IRAMUTEQ na análise de dados em pesquisas qualitativas. Rev. esc. enferm. USP [Internet]. 2018 [acesso em 02 dez 2019]; 52. Disponível em: http://dx.doi.org/10.1590/s1980-220x2017015003353.

10. Salviati ME. Manual do Aplicativo Iramuteq (versão 0.7 Alpha 2 e R Versão 3.2.3). Planaltina. [Internet]. 2017 [acesso em 19 jan 2019]. Disponível em: http://iramuteq.org/documentation/fichiers/anexomanualdo-aplicativo-iramuteq-par-maria-elisabeth-salviati.

11. Bardin L. Análise de Conteúdo. 70. ed. Lisboa: Edições; 2009.

12. Moura RC de M, Pereira TF, Rebouças FJ, Costa C de M, Lernades AMG, Silva LKA da, Rocha K de MM da. Cuidados de enfermagem na prevenção da violência obstétrica. Enferm. Foco [Internet]. 2018 [acesso em 02 dez 2019]; 9(4). Disponível em: https://doi.org/10.21675/2357-707X.2018.v9.n4.1333.

13. Souza TMC, Rezende FF. Violência contra mulher: concepções e práticas de profissionais de serviços públicos. Est. Inter. Psicol. [Internet]. 2018 [acesso em 02 dez 2019]; 9(2). Disponível em: http://pepsic. bvsalud.org/scielo.php?script=sci arttext\&pid=\$2236-64072018000200003.

14. Oliveira MT de, Ferigato SH. A atenção às mulheres vítimas de violência doméstica e familiar: a construção de tecnologias de cuidado da terapia ocupacional na atenção básica em saúde. Cad. Bras. Ter. Ocup. [Internet]. 2019 [acesso em 02 dez 2019]; 27(3). Disponível em: http://dx.doi.org/10.4322/25268910.ctoao1729. 
15. Acosta DF, Gomes VL de O, Oliveira DC de, Marques SC, Fonseca AD da. Representações sociais de enfermeiras acerca da violência doméstica contra a mulher: estudo com abordagem estrutural. Rev. Gaúcha Enferm. [Internet]. 2018 [acesso em 14 fev 2019]. 39. Disponível em: https://doi. org/10.1590/1983-1447.2018.61308.

16. Silva CD, Gomes VL de O, Fonseca AD da, Gomes MT, Arejano CB. Representação da violência doméstica contra a mulher: comparação entre discentes de enfermagem. Rev. Gaúch. Enferm. [Internet]. 2018 [acesso em 21 fev 2019]; 39. Disponível em: http://dx.doi.org/10.1590/1983-1447.2018.63935.

17. Silva LEL da, Oliveira MLC de. Violência contra a mulher: revisão sistemática da produção científica nacional no período de 2009 a 2013. Cien Saude Colet [Internet]. 2015 [acesso em 19 fev 2019]; 20(11). Disponível em: https://doi.org/10.1590/1413-812320152011.11302014.

18. Barbiani R, Nora CRD, Schaefer R. Nursing practices in the primary health care context: a scoping review. Rev. Latino-Am. Enfermagem [Internet]. 2016 [acesso em 22 fev 2019]; 24. Disponível em: http:// dx.doi.org/10.1590/1518-8345.0880.2721.

19. Simoes AV, Machado JC, Soares IGB, Rodrigues VP, Pires VMMM, Penna LHG. Identificação e conduta da violência doméstica contra a mulher sob a ótica dos estudantes universitários. Enfermería Actual de Costa Rica [Internet]. 2019 [acesso em 02 dez 2019]; (37): 95-109. Disponível em: http://dx.doi. org/10.15517/revenf.v0ino.37.35967.

Recebido: 22/03/2019

Finalizado: $28 / 02 / 2020$

\author{
Autor Correspondente: \\ Renata Clemente dos Santos \\ Centro Universitário Unifacisa \\ R. Manoel Cardoso Palhano, 124-152 - 58408-326 - Campina Grande, PB, Brasil \\ E-mail: renata.clemente@hotmail.com
}

Contribuição dos autores:

Contribuições substanciais para a concepção ou desenho do estudo; ou a aquisição, análise ou interpretação de dados do estudo - GKNA

Elaboração e revisão crítica do conteúdo intelectual do estudo - JCVL, JCS, RSCS

Aprovação da versão final do estudo a ser publicado - CMRMS, RQS

Responsável por todos os aspectos do estudo, assegurando as questões de precisão ou integridade de qualquer parte do estudo - RCS 